\title{
ON ALMOST PERIODIC SOLUTIONS FOR UNDAMPED SYSTEMS WITH ALMOST PERIODIC FORCING
}

\author{
GEORGE SEIFERT ${ }^{1}$
}

\begin{abstract}
Using the method of averaging, we give sufficient conditions for the existence of an almost periodic solution of an undamped oscillatory system with almost periodic forcing, and show that these can be applied to a Duffing equation with almost periodic forcing provided the nonlinear term is sufficiently small, and the natural frequency of the linear part of the system is included in the set of Fourier exponents of the forcing function.
\end{abstract}

It is well known that the real linear second order scalar differential equation $x^{\prime \prime}+x=f(t)$, with $f$ almost periodic (a.p. for short), cannot have almost periodic solutions, or even a solution bounded on the real line, whenever 1 is a Fourier exponent of $f$.

It is the purpose of this note to obtain a result which will show that for such so-called resonance cases, there exist sufficiently small perturbations of this equation, involving only functions of $x$ which have a.p. solutions. More precisely, if $f$ has Fourier exponent 1 and one of the corresponding coefficients of the sine or cosine term in the Fourier series is zero, then there exist positive numbers $\nu$ and $\epsilon_{0}=\epsilon_{0}(v)$ such that for $0<\epsilon \leqq \epsilon_{0}$ the equation

$$
x^{\prime \prime}+x-\epsilon \nu x+\epsilon^{3} x^{3}=f(t)
$$

will have an a.p. solution. The magnitude of $v$ is proportional to the absolute value of the Fourier coefficient corresponding to the Fourier exponent 1 in the series for $f$. This a.p. solution is unstable in the sense of exhibiting the so-called saddle-point property, and the suprema of its absolute value and that of its derivative over all real $t$ become unbounded as $\epsilon \rightarrow 0$.

This property of (1) seems interesting for cases where $f$ has a sequence $\left\{\lambda_{k}\right\}, k=1,2, \cdots$, of Fourier exponents, $\lambda_{k}^{2}<1$, and $\lambda_{k}^{2} \rightarrow 1$ as $k \rightarrow \infty$.

Received by the editors February 22, 1971.

AMS 1970 subject classifications. Primary 34C25; Secondary 34C30.

Key words and phrases. Almost periodic functions, undamped oscillatory systems, method of averaging, Duffing equation.

${ }^{1}$ This research was supported in part by the National Science Foundation under Grant GP-24418.

(c) American Mathematical Society 1972 
Thus for any such $\lambda_{k}$ with $1-\lambda_{k}^{2}<\epsilon_{0} \nu$ there exists a positive $\epsilon_{k}=$ $\left(1-\lambda_{k}^{2}\right) / \nu$, for which (1) has an a.p. solution, and yet we have resonance with the linear part of (1). It appears, therefore, that the nonlinearity $\epsilon^{3} x^{3}$ destroys this resonance effect, but that as $\epsilon \rightarrow 0$ the resonance with the $\cos t$ or $\sin t$ term in any case takes over and the a.p. solutions become unbounded, as might be expected.

Our primary tool for obtaining our general result is the method of averaging; cf. [1, pp. 186-190]. In fact, we use a slightly modified version of Theorem 3.1 on p. 190 of this reference; the additional hypothesis on $f(t, x, \epsilon)$ that it be a.p. in $t$ uniformly for $(x, \epsilon)$ in compact subsets of $R^{n} \times$ $[0, \infty)$ seems to be required in the proof of this theorem. ${ }^{2}$ Here, and henceforth, we denote by $R$ the set of all real numbers, by $R^{n}$ the set of all real $n$-vectors, and if $x \in R^{n}$, by $|x|$ a convenient norm in $R^{n}$. We also denote by $m(g(t))$ the mean value of the a.p. function $g(t)$; i.e.,

Our general result is

$$
m(g(t))=\lim _{T \rightarrow+\infty} \frac{1}{T} \int_{0}^{T} g(t) d t .
$$

\section{THEOREM 1. Consider the equation}

$$
x^{\prime}=(A+\epsilon C(t)) x+\epsilon g(x, \epsilon)+\epsilon p(t)
$$

where $x, g(x, \epsilon)$ and $p(t)$ are in $R^{n}, A$ is a $n \times n$ real matrix similar to a diagonal matrix with pure imaginary entries, $C(t)$ and $p(t)$ are a.p., $g$ and its first partials with respect to $x$ are continuous on $R^{n} \times[0, \infty)$, and $\epsilon \geqq 0$. Let

(i) $C_{0}=m\left(e^{-t A} C(t) e^{t A}\right)$,

(ii) $g_{0}(y)=m\left(e^{-t A} g\left(e^{t A} y, 0\right)\right)$, and

(iii) $b_{0}=m\left(e^{-t A} p(t)\right)$,

and suppose the equation

$$
C_{0} y+g_{0}(y)+b_{0}=0
$$

has a solution $\bar{y}$ such that the eigenvalues of $C_{0}+\left(\partial g_{0} / \partial y\right)(\bar{y})$ all have real parts different from zero. Then there exists an $\epsilon_{0}>0$ such that for each $\epsilon, 0 \leqq \epsilon \leqq \epsilon_{0}$, equation (2) has an a.p. solution $x(t, \epsilon)$ such that $\left|x(t, \epsilon)-e^{t A} \bar{y}\right| \rightarrow 0$ as $\epsilon \rightarrow 0$ uniformly for $t \in R$.

Proof. Put $x=e^{t A} y$ and after simplification, (2) becomes

$$
y^{\prime}=\epsilon e^{-t A}\left[C(t) e^{t A} y+g\left(e^{t A} y, \epsilon\right)+p(t)\right]
$$

${ }^{2}$ ADDED IN PROOF. Since this paper was submitted, it has been determined that the additional hypothesis to Theorem 3.1 in [1] need only be that $f(t, x, \epsilon) \rightarrow f(t, x, 0)$ as $\epsilon \rightarrow 0$ uniformly for $(t, x)$ in $R \times K, K$ any compact set in $R^{n}$. 
We now average the right side of (3) and obtain $y^{\prime}=\epsilon\left[C_{0} y+g_{0}(y)+b_{0}\right]$ and the theorem now follows immediately from our hypotheses and Theorem 3.1, p. 190, in [1], suitably amended as above; it is important to observe that $e^{t A}$ is a.p.

By simple changes of variables, certain other equations can be put into the form of (2). For example, consider

$$
y^{\prime}=(A+\epsilon C(t)) y+h(y)+\epsilon^{2} p(t)
$$

where $A, C, p$ are as above, $h$ has continuous first partials on $R^{n}$ and satisfies $h(y)=O\left(|y|^{2}\right)$ as $|y| \rightarrow 0$, and $\epsilon>0$. If we put $y=\epsilon x$, then $h(\epsilon x)=\epsilon^{2} g(y, \epsilon)$ and (4) becomes, after dividing by $\epsilon$,

$$
x^{\prime}=(A+\epsilon C(t)) x+\epsilon g(y, \epsilon)+\epsilon p(t)
$$

which is in the form of (2).

Also if $C, A$, and $p$ are as above, and $h$ is a polynomial in the components of $x$ of degree $m \geqq 1$, then the equation

$$
y^{\prime}=(A+\epsilon C(t)) y+\epsilon^{m} h(y)+p(t), \quad \epsilon>0,
$$

can be put into the form of (2) by the change of variable $y=x / \epsilon$, and multiplying the resulting equation by $\epsilon$. We note that here $g(y, 0)=$ $\lim _{\epsilon \rightarrow 0} \epsilon^{m} h(y / \epsilon)$ depends only on the coefficients of the terms of degree $m$ in the polynomial $h$, and that (6) can be regarded as a certain perturbation of the linear system $y^{\prime}=A y+p(t)$, where no conditions are placed on $\sup |p(t)|, t \in R$.

Using this last change of variables, we show that equation (1) can be considered in terms of an equivalent system of the form of (2). Let the real-valued a.p. $f$ in (1) have Fourier series

$$
f \sim \sum_{j=1}^{\infty} a_{j} \cos \lambda_{j} t+b_{j} \sin \lambda_{j} t, \quad \lambda_{1}=1 .
$$

Multiplying (1) by $\epsilon>0$, putting $\epsilon x=y$, we obtain

$$
y^{\prime \prime}+y-\epsilon \nu y+\epsilon y^{3}=\epsilon f(t),
$$

and consider the usual equivalent system, defined also for $\epsilon=0$ :

$$
y_{1}^{\prime}=y_{2}, \quad y_{2}^{\prime}=-y_{1}+\epsilon\left(v y_{1}-y_{1}^{3}+f(t)\right) .
$$

With

and

$$
A=\left(\begin{array}{cc}
0 & 1 \\
-1 & 0
\end{array}\right), \quad C(t)=\left(\begin{array}{ll}
0 & 0 \\
\nu & 0
\end{array}\right), \quad g=-\left(\begin{array}{c}
0 \\
y_{1}^{3}
\end{array}\right)
$$

$$
p(t)=\left(\begin{array}{c}
0 \\
f(t)
\end{array}\right)
$$


we compute

$$
\begin{aligned}
C_{0} & =\frac{1}{2}\left(\begin{array}{cc}
0 & -v \\
v & 0
\end{array}\right), \\
g_{0}\left(y_{1}, y_{2}\right) & =\left(\begin{array}{cc}
3\left(y_{1}^{2}+y_{2}^{2}\right) & y_{2} / 8 \\
-3\left(y_{1}^{2}+y_{2}^{2}\right) & y_{1} / 8
\end{array}\right), \\
b_{0} & =\frac{1}{2}\left(\begin{array}{c}
-b_{1} \\
a_{1}
\end{array}\right),
\end{aligned}
$$

and equation (2.1) becomes essentially the system

$$
\begin{aligned}
-4 v y_{2}+3\left(y_{1}^{2}+y_{2}^{2}\right) y_{2}-4 b_{1} & =0 \\
4 v y_{1}-3\left(y_{1}^{2}+y_{2}^{2}\right) y_{1}+4 a_{1} & =0 .
\end{aligned}
$$

Suppose $b_{1}=0, a_{1}>0$. We seek a solution of (9) with $y_{2}=0$; i.e., we seek solutions of

$$
-4 v y_{1}+3 y_{1}^{3}=4 a_{1} \text {. }
$$

If $v>\left(9 a_{1} / 2\right)^{2 / 3}$, then this equation has 3 real roots, one of which, say $\bar{y}_{1}$, satisfies $\bar{y}_{1}<0,4 v / 9<\bar{y}_{1}^{2}<4 v / 3$. We have, taking $\bar{y}=\left(\bar{y}_{1}, 0\right)$,

$$
C_{0}+\frac{\partial g_{0}}{\partial y}(\bar{y})=\left(\begin{array}{cc}
0 & -v / 2+3 \bar{y}_{1}^{2} / 8 \\
\nu / 2-9 \bar{y}_{1}^{2} / 8 & 0
\end{array}\right) .
$$

Since the eigenvalues of this matrix are real, distinct from zero (in fact, of opposite sign), we can apply Theorem 1 and conclude the existence of an a.p. solution of (1) for $\epsilon>0$ and sufficiently small. Clearly the cases $a_{1}<0, b_{1}=0$, and $a_{1}=0,\left|b_{1}\right| \neq 0$ will yield, upon similar treatment, the same conclusion. Thus we have essentially proved

THEOREM 2. Let $a_{1} b_{1}=0,\left|a_{1}+b_{1}\right|=\mu>0$, and $\nu$ any fixed number satisfying $\nu>(9 \mu / 2)^{2 / 3}$. Then there exists $\epsilon_{0}=\epsilon_{0}(\nu)>0$, such that for $0<\epsilon \leqq \epsilon_{0}$, equation (1) has an a.p. solution $x(t, \epsilon)$ with saddle-point type stability. Further, $\epsilon x(t, \epsilon)-\left(\bar{y}_{1} \cos t+\bar{y}_{2} \sin t\right) \rightarrow 0$ as $\epsilon \rightarrow 0$ where $\bar{y}_{1} \neq 0$ is as above and $\bar{y}_{2}=0$ if $a_{1} \neq 0, b_{1}=0$, while $\bar{y}_{2} \neq 0, \bar{y}_{1}=0$ in case $a_{1}=0, b_{1} \neq 0$.

It is clear that the same existence theorem can be obtained for a nonautonomous equation of the form of (1) with $v$ replaced by $v+q(t), q(t)$ any real-valued a.p. function not having 2 as a Fourier exponent. This true because in that case the matrix $C_{0}$ turns out to be the same as before.

The saddle-point type stability of the a.p. solution $x(t, \epsilon)$ of (1) follows from the fact that the eigenvalues of the matrix (11) are real and of opposite sign; for further details, cf. [1]. 
Finally, we remark that the condition in Theorem 2 that either $a_{1}$ or $b_{1}$ be zero is not really required; if both are nonzero, we can clearly find a number $\omega$ such that $f(t+\omega)$ will have a Fourier series where either $a_{1}$ or $b_{1}$ is zero. We may then use the new time variable $\tau=t+\omega$ and obtain the same conclusion, since clearly $x(t)$ is a.p. if and only if $x(t+\omega)$ is.

\section{REFERENCE}

1. J. K. Hale, Ordinary differential equations, Wiley, New York, 1969.

Department of Mathematics, Iowa State University, Ames, Iowa 50010 